\title{
High self-reactivity drives T-bet and potentiates Treg function in tissue- specific autoimmunity
}

\author{
Maran L. Sprouse, ${ }^{1}$ Marissa A. Scavuzzo, ${ }^{2}$ Samuel Blum, ${ }^{1}$ Ivan Shevchenko, ${ }^{1}$ Thomas Lee, ${ }^{1}$ \\ George Makedonas, ${ }^{3}$ Malgorzata Borowiak, ${ }^{4,5}$ Matthew L. Bettini,, ${ }^{1,5}$ Maria Bettini'1,5
}

Department of Pediatrics, Section of Diabetes and Endocrinology, ${ }^{2}$ Program in Developmental Biology, ${ }^{3}$ Department of Pediatrics, Center for Human Immunobiology, ${ }^{4}$ Department of Molecular and Cellular Biology, Center for Cell and Gene Therapy, and ${ }^{5}$ McNair Medical Institute, Texas Children's Hospital, Baylor College of Medicine, Houston, Texas, USA.

T cell receptor (TCR) affinity is a critical factor of Treg lineage commitment, but whether selfreactivity is a determining factor in peripheral Treg function remains unknown. Here, we report that a high degree of self-reactivity is crucial for tissue-specific Treg function in autoimmunity. Based on high expression of CD5, we identified a subset of self-reactive Tregs expressing elevated levels of T-bet, GITR, CTLA-4, and ICOS, which imparted significant protection from autoimmune diabetes. We observed that T-bet expression in Tregs, necessary for control of Th1 autoimmunity, could be induced in an IFN $\gamma$-independent fashion and, unlike in conventional T cells (Tconv), was strongly correlated with the strength of TCR signaling. The level of CD5 similarly identified human Tregs with an increased functional profile, suggesting that $C D 5^{\text {hi }}$ Tregs may constitute an efficacious subpopulation appropriate for use in adoptive Treg therapies for treatment of inflammatory conditions. Overall, this work establishes an instrumental role of high TCR self-reactivity in driving Treg function.

Conflict of interest: The authors have declared that no conflict of interest exists.

Submitted: September 6, 2017 Accepted: December 14, 2017 Published: January 25, 2018

Reference information: JCI Insight. 2018;3(2):e97322. https:// doi.org/10.1172/jii.insight.97322.

\section{Introduction}

Tregs are multifunctional and heterogeneous lymphocytes important for maintaining immune homeostasis and preventing autoimmunity (1-3). The type of suppressive and regulatory mechanisms utilized by Tregs is often dictated by the tissue site and the type of immune response (4). Accordingly, Tregs exhibit unique $\mathrm{T}$ cell receptor (TCR) repertoires depending on the tissue site examined, suggesting that immune regulation in different anatomical locations is tissue specific $(2,5)$. Several studies suggest that TCR specificity is a critical feature of Tregs in preventing tissue-specific autoimmunity (6-9); however, within the Treg population, there exists significant variability of TCR self-reactivity (10-12). Thus, it remains unclear whether the level of TCR affinity for self-antigen is an important determinant of Treg function during a tissue-specific response.

TCR affinity is a principal determinant of Treg lineage commitment during thymocyte development, as Tregs generally express TCRs with stronger self-reactivity in comparison with conventional $\mathrm{T}$ cells (Tconvs) (10, 13-16). Moreover, TCR signaling is continually required for Treg-suppressive function in the periphery (17). While it is still unknown to what degree TCR affinity is important for Treg function, based on our previous observations, we suspect that a certain high level of self-reactivity is more important for Treg functional capacity than for Treg development. When tissue-specific autoimmune TCRs of either high or low affinity were expressed in TCR retrogenic mice, both high- and low-affinity TCRs supported Treg development (18). Remarkably, only mice expressing the high-affinity insulin-specific TCRs experienced accelerated diabetes development upon Treg deletion, which indicated a role for TCR affinity in Treg function. Together, these observations led us to hypothesize that, within a relatively wide range of self-reactivity, highly self-reactive Tregs are more functional during tissue-specific autoimmunity.

This study addresses how the level of TCR self-reactivity impacts Treg function. We focused our analysis on tissue-infiltrating Tregs and assessed their suppressive phenotype and function in the mouse model of type 1 diabetes. Our findings establish a critical role for TCR self-reactivity during autoimmunity, where strong TCR signaling in response to self-ligands leads to generation of optimal Treg responses. 
A

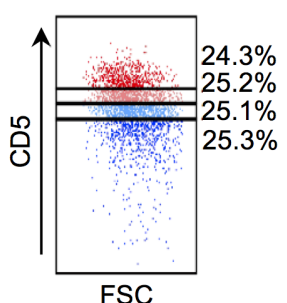

FSC

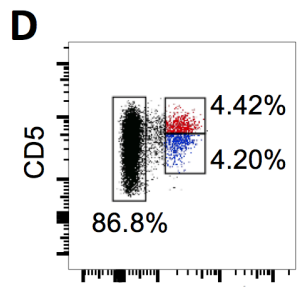

Foxp3-GFP

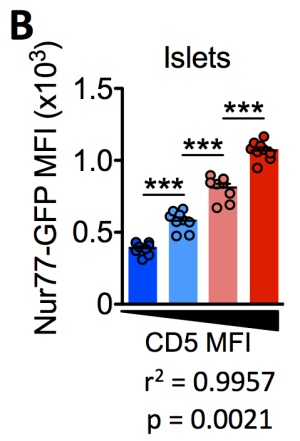

C

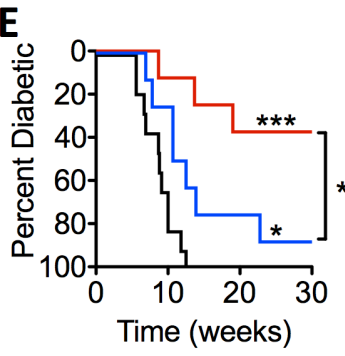

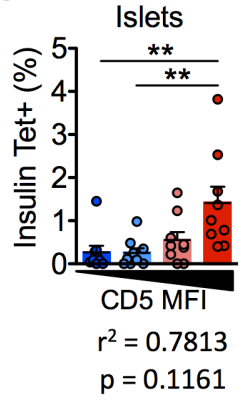

F Islets

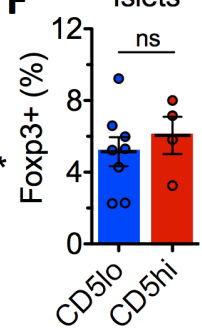

Figure 1. CD5 correlates with self-reactivity and marks highly functional Tregs in autoimmunity. (A) Representative flow plot of CD5 quartile gating. (B and C) Analysis of 7- to 12-week-old NOD.Nur77 ${ }^{\text {CFP }}$ female mice. An average of 9 mice from 1 experiment is shown. (B) Correlation between CD5 expression and GFP reporter of Nur77 expression in islet-infiltrating Tregs $\left(\mathrm{CD}^{+}{ }^{+} \mathrm{CD3}^{+}{ }^{+} \mathrm{Fxp3}^{+}\right)$. (C) Correlation between CD5 expression and InsB:923 tetramer staining of islet-infiltrating Tregs (CD4+CD3+Foxp3 $\left.{ }^{+}\right)$. Gating strategy depicted in Supplemental Figure 1C. (D) Representative gating strategy for sorting Teff (CD4+CD5+CFP-) and Tregs (CD4 $\left.{ }^{+} C D 5^{+} C F P^{+}\right)$from the islets of NOD.Foxp3 ${ }^{\mathrm{CFP}}$ mice. (E) Diabetes incidence of NOD. $\mathrm{Cra}^{-/-}$mice which received 16,000 Teff alone ( $n=10$, black line), in combination with 4,000 CD5 $^{\text {hi }}$ Tregs ( $n=8$, red line), or with 4,000 CD5 $5^{10}$ Tregs ( $n=8$, blue line). Mice were monitored for diabetes development for 30 weeks. Data are pooled from 2 independent experiments. (F) Frequency of Tregs in the islets of NOD. $\mathrm{Cra}^{-/-}$recipient mice at end point. Data are pooled from 4 ( $\left.C D 5^{\text {hi }}\right)$ and $8\left(\mathrm{CD}^{10}\right)$ mice. Significance was determined by 1-way ANOVA with Bonferroni's multiple-comparisons test (B and $\mathbf{C})$ log-rank test $(\mathbf{E})$, and Mann-Whitney $U$ test $(\mathbf{F})$. The mean \pm SEM is shown. $(P>0.05)$, ${ }^{*} P<$ $0.05,{ }^{* *} P<0.005,{ }^{* * *} P<0.0005$.

\section{Results}

CD5 is a faithful marker of self-reactivity. CD5 is upregulated on T cells during thymic development in proportion to the strength of TCR signaling received in response to self-ligands, and it is stably maintained at a set level in the periphery $(18,19)$. Although, CD5 is considered to be the most faithful marker of self-reactivity described to date, specifically in the Tconv population, direct correlation between CD5 and the magnitude of Treg self-reactivity in tissue autoimmunity has not been assessed $(20,21)$. Comparison of CD5 levels on Tconv $\left(\mathrm{CD}^{+}{ }^{+} \mathrm{CD}^{+}{ }^{+} \mathrm{Foxp}^{-}\right)$and Treg $\left(\mathrm{CD}^{+}{ }^{+} \mathrm{CD} 3^{+} \mathrm{Foxp}^{+}\right)$cells confirmed previous observations that Tregs express higher levels of CD5, indicative of their general propensity for self-reactivity (Supplemental Figure 1A; supplemental material available online with this article; https://doi.org/10.1172/ jci.insight.97322DS1) (22). CD5 expression was higher on islet-infiltrating Tconvs and Tregs compared with cells obtained from peripheral draining lymph nodes (Supplemental Figure 1A). This indicated that self-reactive $\mathrm{T}$ cells were recruited to the pancreatic islets, which are sites of tissue-specific autoimmunity in NOD mice. Similar to CD5, TCR signaling (Nur77 $7^{\mathrm{GFP}}$ ) and frequency of tetramer-positive cells were higher in islet-infiltrating Tregs compared with Tconvs (Supplemental Figure 1, B-D). When we subdivided the islet-infiltrating Foxp3 $3^{+}$Treg population into 4 equal groups based on CD5 expression (Figure 1A), we observed a near linear correlation $\left(r^{2}=0.9957\right)$ of CD5 with the level of TCR signaling (Nur77 ${ }^{\text {GFP }}$ ) (Figure $1 \mathrm{~B})$, as well as a strong correlation $\left(\mathrm{r}^{2}=0.7813\right)$ with insulin tetramer staining (Figure $\left.1 \mathrm{C}\right)$. The correlation between CD5 and Nur77 ${ }^{\mathrm{GFP}}$ or CD5 and tetramer staining was also observed in effector T cells (Teffs), albeit the overall expression values were lower compared with Tregs (Supplemental Figure 1E). To rule out the possibility that CD5 levels were merely a reflection of TCR expression levels, we assessed the level of cell-surface CD3, which was similar across the populations (Supplemental Figure 1F). Therefore, Tregs recruited to the autoimmune tissue exhibited increased yet varied levels of self-reactivity, which was directly correlated with the level of CD5 expression.

Highly self-reactive Tregs impart significant protection from diabetes development. To elucidate the role of TCR affinity in Treg function during autoimmunity, we assessed the ability of highly self-reactive (CD5 ${ }^{\text {hi }}$ ) or mildly self-reactive $\left(C D 5^{10}\right)$ Tregs to prevent diabetes induction by $C D 4^{+}$Teffs. We FACS sorted polyclonal Teffs $\left(\mathrm{CD}^{+} \mathrm{GFP}^{-}\right)$directly from the infiltrated pancreatic islets of NOD.Foxp3 ${ }^{\mathrm{GFP}}$ reporter mice, along with $\mathrm{CD}^{\text {hi }}$ or $\mathrm{CD}^{\text {lo }}$ Tregs $\left(\mathrm{CD}^{+} \mathrm{GFP}^{+}\right)$. The Treg population was divided equally into the top $50 \%\left(\mathrm{CD} 5^{\text {hi }}\right)$ and bottom $50 \%\left(\mathrm{CD}^{10}\right)$ of $\mathrm{CD} 5$ expression (Figure $1 \mathrm{D}$ and Supplemental Figure 2, A and B). Teffs alone, or in combination with either $\mathrm{CD} 5^{\mathrm{hi}}$ or $\mathrm{CD} 5^{\text {1o }}$ Tregs, were transferred into NOD. Tcro ${ }^{-/}$recipients, and mice were monitored for diabetes development. As expected, all mice that received Teffs alone rapidly developed diabetes with $100 \%$ penetrance by 13 weeks after transfer (Figure $1 \mathrm{E}$ ). Surprisingly, CD5 ${ }^{\text {hi }}$ and CD5 $^{\text {lo }}$ Tregs exhibited significantly different protective capacities. Nearly $90 \%$ of mice that received Teffs in combination 

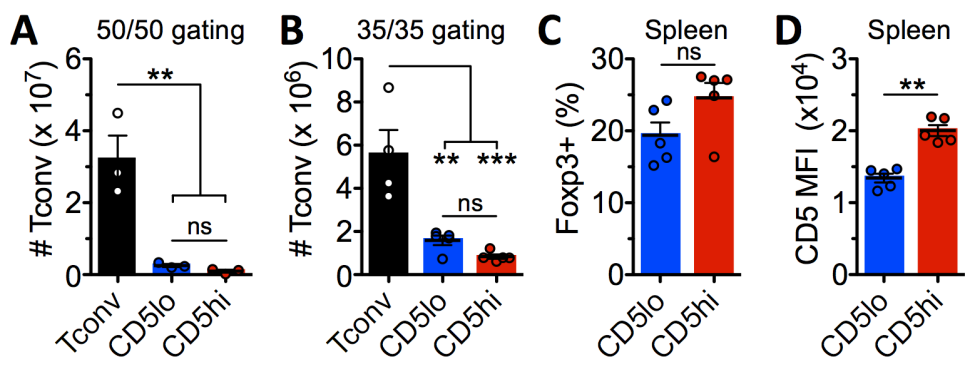

Figure 2. Both CD5 ${ }^{\text {hi }}$ and $\mathrm{CD}^{\text {lo }}$ Tregs can maintain T cell homeostasis. Analysis of NOD.scid recipient spleens 4 weeks after T cell transfer. (A) Mice received $5 \times 10^{5}$ naive Tconv alone (white), in combination with $1.25 \times 10^{5} \mathrm{CD}^{\text {hi }}$ (top 50\%) Tregs (red), or with $1.25 \times 10^{5} \mathrm{CD}^{10}$ (bottom $50 \%$ ) Tregs (blue). Gating strategy and purity depicted in Supplemental Figure $2, C$ and $D$. Tconv expansion is based on total number of $C D 4^{+} C D 3^{+}$Foxp3 $3^{-}$cells in spleens of recipient mice. An average of 3 mice from 1 experiment is shown. (B-D) Mice received $5 \times 10^{5}$ naive Tconv alone, in combination with 1.25 $\times 10^{5} \mathrm{CD}^{\text {hi }}$ (top 35\%) Tregs (red), or with $1.25 \times 10^{5} \mathrm{CD}^{10}$ (bottom 35\%) Tregs (blue). Gating strategy and purity shown in Supplemental Figure 2, F and G. An average of 4-5 mice from 2 experiments is shown. (B) Tconv expansion is based on total number of $\mathrm{CD}^{+} \mathrm{CD}^{+} \mathrm{Foxp3}^{-}$cells in spleens of recipient mice. (C) Frequency of Tregs within the $\mathrm{CD4}^{+} \mathrm{CD}^{+}$population 4 weeks after transfer. (D) CD5 expression in splenic Tregs 4 weeks after transfer. Significance was determined by 1-way ANOVA with Bonferroni's multiple-comparisons test (A and $\mathbf{B})$ and Mann-Whitney $U$ test (C and $\mathbf{D})$. The mean \pm SEM is shown. $(P>0.05),{ }^{* *} P<0.005,{ }^{* *} P<0.0005$.

with $C D 5^{10}$ Tregs were diabetic by 22 weeks after transfer (Figure 1E). Conversely, mice that received Teffs along with $C D 5^{\text {hi }}$ Tregs were largely protected from diabetes development, with less than $40 \%$ of mice developing disease (Figure 1E). The enhanced protective capacity of $\mathrm{CD} 5^{\text {hi }}$ Tregs did not result from a difference in trafficking ability of $\mathrm{CD} 5^{\text {hi }}$ and $C D 5^{\text {lo }}$ Tregs, as both Treg populations were present in the islets at similar frequencies (Figure 1F), indicating functional insufficiency of the $\mathrm{CD} 5^{\text {lo }}$ Tregs. Together, these data suggest that high self-reactivity is associated with improved Treg function during autoimmunity.

Both $C D 5^{\text {hi }}$ and $C D 5^{\text {lo }}$ Tregs maintain $T$ cell homeostasis. We next sought to assess the importance of Treg self-reactivity in an antigen-independent setting. Recent studies have shown that maintenance of immune homeostasis by Tregs is a TCR-dependent event (23). As control of T cell homeostasis in peripheral lymphoid organs can be considered an antigen-independent response, we wanted to address if the level of self-reactivity correlated with Treg ability to maintain immune homeostasis. Naive $\mathrm{T}$ cells $\left(\mathrm{CD} 4^{+} \mathrm{C}\right.$ $\left.\mathrm{D} 45 \mathrm{RB}^{\mathrm{hi}} \mathrm{CD} 25^{-}\right)$were sorted from the spleens of NOD.CD45.2 mice and transferred together with CD $5^{\text {hi }}$ or $\mathrm{CD} 5^{\text {lo }}$ congenically marked Tregs $\left(\mathrm{CD} 4^{+} \mathrm{GFP}^{+} \mathrm{CD} 45.1^{+}\right)$from NOD.Foxp $3^{\text {GFP }}$ mice at a $4: 1$ ratio into NOD.scid recipients. The Treg population was divided equally into the top $50 \%\left(C D 5^{\text {hi }}\right)$ and bottom $50 \%$ $\left(C D 5^{1 \circ}\right)$ of $C D 5$ expression (Supplemental Figure 2, C and D). As expected, mice that received naive T cells alone had robust expansion of Tconvs, with an observed 60-fold increase (Figure 2A). Interestingly, cotransfer of either Treg population resulted in an effective control of naive $\mathrm{T}$ cell expansion (Figure 2A). Even when we further segregated the Treg population into the very top $35 \%\left(C D 5^{\text {hi }}\right)$ and bottom $35 \%\left(\mathrm{CD}^{10}\right)$ (Supplemental Figure 2, F and G), both Treg subsets were still able to control naive $\mathrm{T}$ cell expansion, albeit there was a trend toward somewhat higher Tconv proliferation in the $\mathrm{CD} 5^{\text {10 }}$ Treg group (Figure 2B). Moreover, both Treg subsets maintained similar levels of Foxp3 expression, as well as the transfer Tconv/Treg ratio of 4:1 (Supplemental Figure 2, E and H, and Figure 2C). Importantly, relative levels of $\mathrm{CD} 5$ expression by $\mathrm{CD} 5^{\mathrm{hi}}$ and $\mathrm{CD} 5^{\text {to }}$ Tregs were maintained after transfer (Figure 2D), indicating that $\mathrm{CD} 5$ expression on $\mathrm{T}$ cells is stable. Collectively, these data demonstrate that TCR self-reactivity is not an important determinant of Treg function in an antigen-independent response, as both populations were able to maintain immune homeostasis.

Islet-infiltrating $C D 5^{\text {hi }}$ and $C D 5^{\text {to }}$ Tregs are transcriptionally distinct subsets. To gain insight into the underlying mechanisms of the enhanced suppressive function of $\mathrm{CD} 5^{\text {hi }}$ Tregs in islet autoimmunity, we analyzed the transcriptional profile of Tregs sorted from the spleen and infiltrated pancreatic islets of 12- to 16-week-old NOD.Foxp3 ${ }^{\mathrm{GFP}}$ reporter mice. Principle component analysis (PCA) of splenic Tregs, islet Tregs, and islet Teffs showed that the 2 spleen-derived Treg subsets were similar (Figure $3 \mathrm{~A}$ ), while CD5 $5^{\text {10 }}$ islet-derived Tregs were distinct from splenic Tregs but unable to fully attain the transcriptional landscape associated with enhanced suppressive function of CD5 $5^{\text {hi }}$ Tregs (Figure $3 A$ ). Further analysis of the $C D 5^{\text {hi }}$ and $\mathrm{CD} 5^{10}$ Tregs from the islets showed differential expression of over 2,700 genes $(P<0.05)$ (Figure 3B). 
A

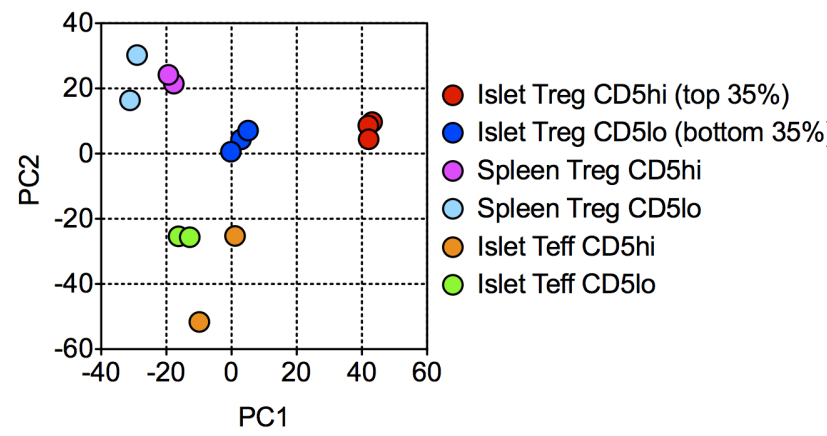

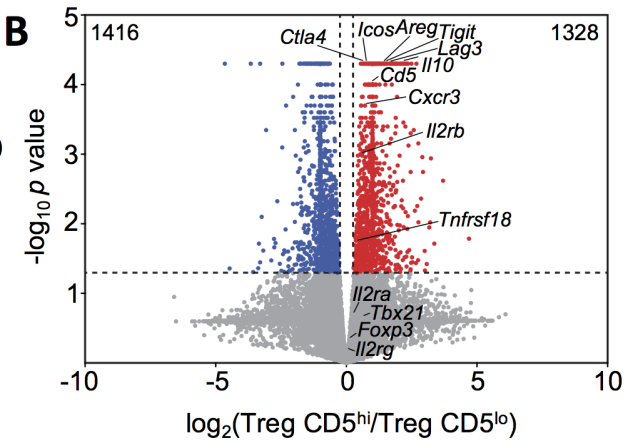

C

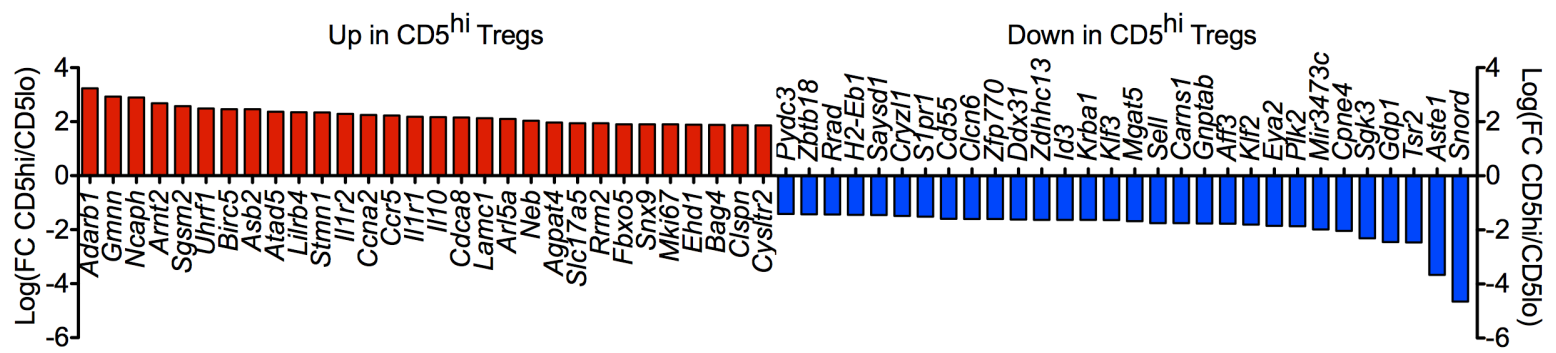

D
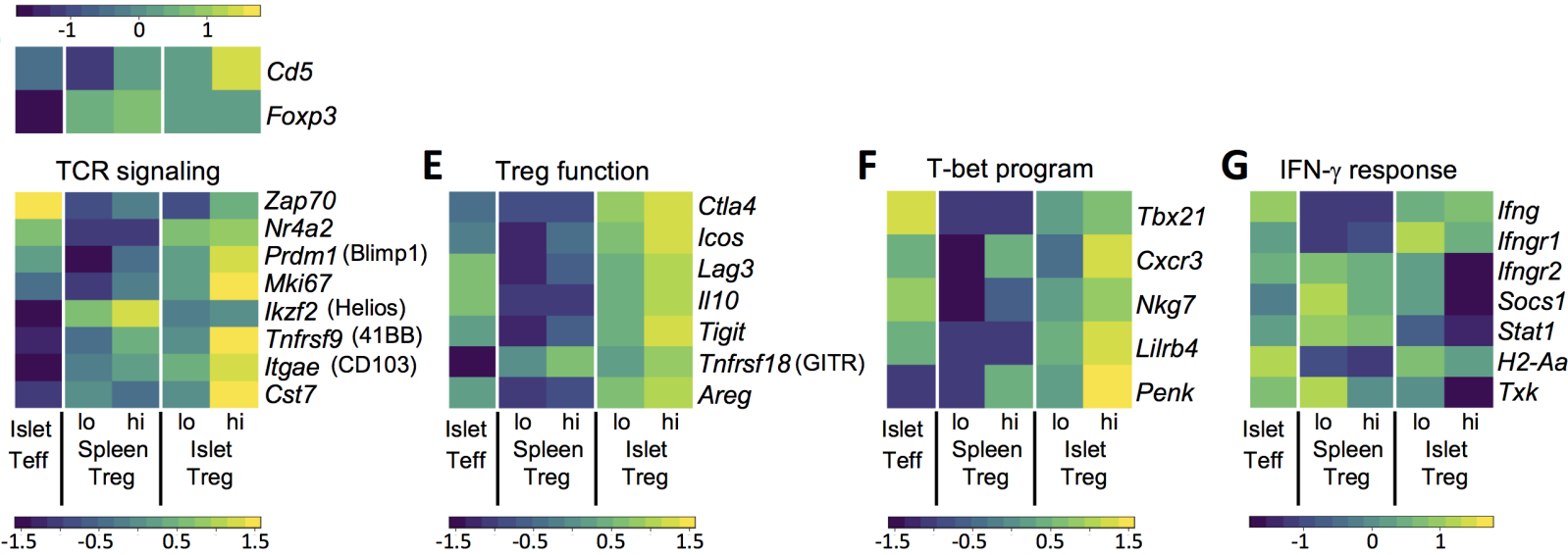

Figure 3. Transcriptional landscape of $\mathbf{C D} 5^{\text {hi }}$ and $C D 5^{10}$ Tregs. (A) Principle component analysis. (B) Volcano plot for CD5 ${ }^{\text {hi }}$ (red) vs. CD5 ${ }^{10}$ (blue) isletinfiltrating Treg comparison. $-\log _{10} P$ value is plotted against the fold change $\left(\log _{2}\right.$ scale). Dashed horizontal line corresponds to $P<0.05$. Numbers indicate the number of genes differentially expressed. (C) Top 30 up- and downregulated genes $(q<0.05)$ in CD5 hi compared with CD5 ${ }^{10}$ islet Tregs. (D-G) $\log _{2} Z$ score normalized RNA-seq expression values of genes are represented as a heatmap. Genes had a pairwise difference with FDR $<0.05$ for at least 1 comparison.

Among the 1,328 genetic transcripts increased in CD5 ${ }^{\text {hi }}$ Tregs, several were associated with Treg-suppressive function, including those previously described to be critical for suppressing islet autoimmunity, Icos (24), Illo (25), and Ctla4 (26) (Figure 3B). The 30 most increased and decreased genetic transcripts in CD5 ${ }^{\text {hi }}$ Tregs $(q<0.05)$ included higher levels of the hallmark suppressive cytokine $I l 10$ and proliferation genes Mki67 and Uhrf1 (27), along with decreased expression of negative regulators of TCR clustering Gbp1 (28) and Mgat5 (29) (Figure 3C). In line with the idea that TCR signaling strength is driving the transcriptional difference between $\mathrm{CD} 5^{\text {hi }}$ and $\mathrm{CD} 5^{\text {lo }}$ islet Tregs, $\mathrm{CD} 5^{\text {hi }}$ Tregs from the islets showed a significant increase in expression of genes associated with TCR signaling (Zap 70), as well as genes regulated by Nur77 directly downstream of TCR activation (Helios, Tnfrsf9, Itgae, Cst7) (30) (Figure 3D). Moreover, many of the genes associated with Treg-suppressive function that were upregulated in CD5 ${ }^{\text {hi }}$ islet Tregs were previously shown to be under the control of TCR (Ctla4, Icos, Lag3, Il10, Tigit) (Figure 3E) (17, 31). Interestingly, $C D 5^{\text {hi }}$ islet Tregs had increased expression of Areg (Figure 3E), a Treg effector molecule involved in tissue repair (3), perhaps indicating that highly self-reactive Tregs do not only suppress the ongoing autoimmune response but also aide in tissue repair. Overall, this suggests that high TCR self-reactivity drives Treg functional potential in the islets. 

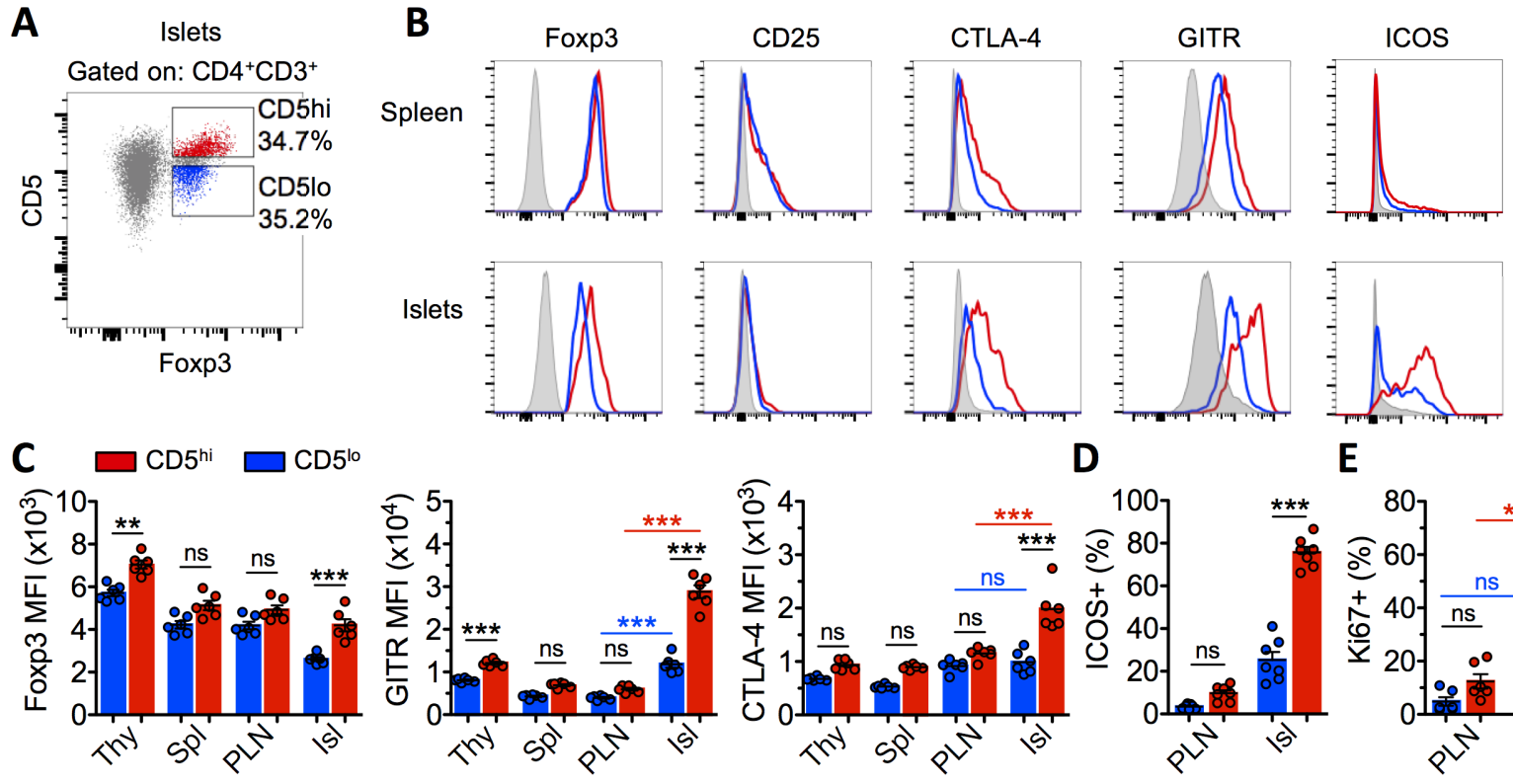

D

E
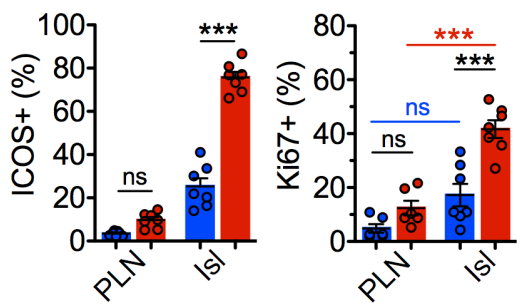

Figure 4. $C D 5^{\text {hi }}$ Tregs constitute a functionally distinct subset. Analysis of 10- to 18- week-old female NOD mice. (A) Representative gating of $\mathrm{CD4}^{+} \mathrm{CD}^{+}{ }^{+} \mathrm{Foxp3}^{+} \mathrm{CD}^{\mathrm{hi}}$ or CD5 ${ }^{10}$ Tregs. (B) Representative expression of Foxp3, CD25, CTLA-4, GITR, and ICOS on CD5 ${ }^{\text {hi }}$ (red) or CD5 $5^{10}$ (blue) Tregs from the spleen (top) or infiltrated islets (bottom). (C) Quantification of Foxp3, GITR, and CTLA-4 expressed by CD5 $5^{\text {hi }}$ or CD $5^{10}$ Tregs from the thymus (Thy), spleen (Spl), pancreatic LN (PLN), and islets (|sI). An average of 6 mice from 2 experiments is shown. (D) Quantification of ICOS expressed by CD5 hi or CD5 ${ }^{10}$ Tregs from the PLN and Isl. An average of 7 mice from 2 experiments is shown. (E) Frequency of Ki67 $7^{+}$CD5 ${ }^{\text {hi }}$ or CD5 ${ }^{10}$ Tregs from the PLN or infiltrated IsI. An average of 7 mice from 3 experiments is shown. Significance was determined by 1-way ANOVA with Bonferroni's multiple-comparisons test (C-E). The mean \pm SEM is shown. $(P>0.05),{ }^{* *} P<0.005,{ }^{* * *} P<0.0005$.

Recent studies have shown that Tregs expressing T-bet and CXCR3 are indispensable for the control of Th1 immune responses, including autoimmune diabetes $(31,32)$. Interestingly, CD $5^{\text {hi }}$ Tregs expressed significantly higher levels of genes regulated by T-bet, including Cxcr3 (Figure 3F). Somewhat paradoxically, gene ontology analysis revealed that $\mathrm{CD} 5^{\text {hi }}$ Tregs from the islets had decreased expression of genes involved in the response to IFN $\gamma$ (Figure 3G and Supplemental Figure 3), which has previously been described to be necessary for T-bet expression by Tregs (33). This might suggest that the transcriptional program necessary for optimal Treg function in tissue-specific autoimmunity relies primarily on TCR signaling rather than inflammatory cues.

$C D 5^{\text {hi }}$ Tregs constitute a phenotypically distinct subset. The transcriptional landscape of CD5 ${ }^{\text {hi }}$ Tregs was enriched for genes associated with Treg function. To confirm these observations at the protein level, we analyzed the expression of key markers associated with Treg lineage stability and function: Foxp3, CD25, GITR, CTLA-4, and ICOS (24, 34-38). Similar to the RNA sequencing (RNA-seq) analysis, the Treg population was divided equally into the top $35 \%\left(C D 5^{\text {hi }}\right)$ and bottom $35 \%\left(C D 5^{10}\right)$ of $\mathrm{CD} 5$ expression (Figure $\left.4 \mathrm{~A}\right)$. Analysis of Foxp3 revealed a significant increase in $\mathrm{CD} 5^{\text {hi }}$ islet Tregs, perhaps suggesting enhanced lineage stability (Figure 4, B and C). CD25 expression was modestly increased among the CD $5^{\text {hi }}$ Tregs (Figure 4B and Supplemental Figure 4A). Expression of CTLA-4, GITR, and ICOS, on the other hand, were significantly higher in CD5 $5^{\text {hi }}$ Tregs (Figure 4, B-D). This phenotypic difference between the 2 Treg subsets was also observed in spleens of the nonautoimmune prone C57BL/6 strain (Supplemental Figure 4, B-D), suggesting that the difference in these Treg subsets is not due to ongoing autoimmunity or limited to a particular strain. Consistent with transcriptome analysis, we observed enhanced proliferation of islet-infiltrating CD $5^{\text {hi }}$ Tregs, as indicated by a significantly higher percent of Ki6 $7^{+}$cells $\left(17 \% \mathrm{Ki}^{+} 7^{+} \mathrm{CD} 5^{\text {lo }}\right.$ compared with $\left.42 \% \mathrm{CD} 5^{\text {hi }}\right)$ (Figure 4E). KLRG1, a marker of terminally differentiated Tregs (39), in combination with high ICOS expression has recently been associated with Treg plasticity in the islets of NOD mice (40). While a higher frequency of CD $5^{\text {hi }}$ Tregs expressed KLRG1, the KLRG1+ $1^{+}$populations in the islets were relatively low ( $2 \%$ for CD $5^{\text {lo }}$ Tregs and $4 \%$ for $\mathrm{CD}^{\mathrm{hi}}$ Tregs) (Supplemental Figure 4E), suggesting that Treg lineage instability was not responsible for the decrease in $\mathrm{CD}^{\text {lo }}$ Treg function. Together, these observations suggest that highly self-reactive Tregs represent a more functionally poised population, and the differences are amplified during tissue-specific responses. 


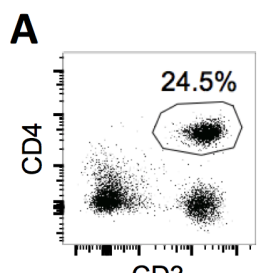

CD3

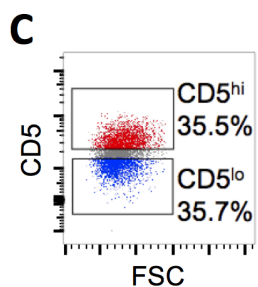

FSC

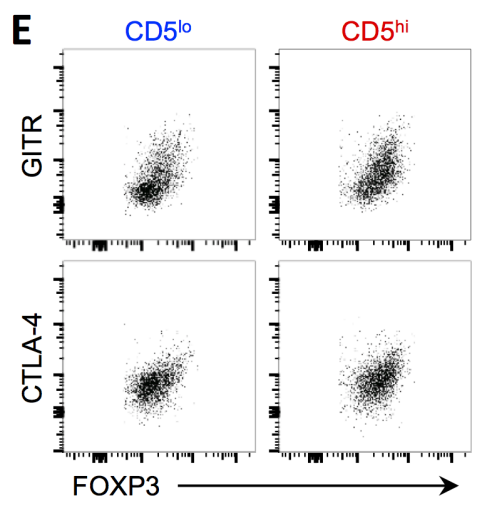

Gating strategy for

B CD127loCD25+FOXP3+ Tregs

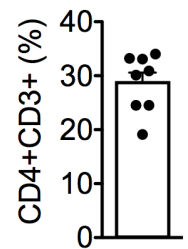

D

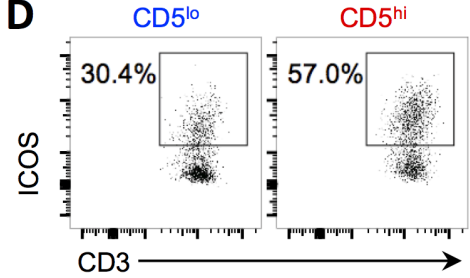

$\mathbf{F}$
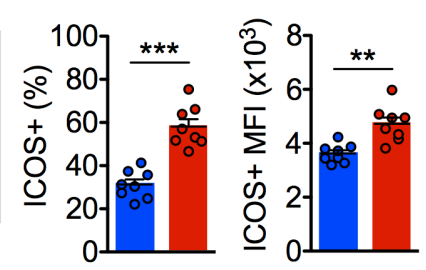

Figure 5. Human $\mathrm{CD} 5^{\text {hi }}$ Tregs display an enhanced functional profile. Analysis of fresh human peripheral blood T cells. An average of 8 healthy subjects from 1 experiment is shown. (A) Representative gating strategy of human peripheral blood T cells ( $\left.C D 4^{+} \mathrm{CD}^{+}\right)$. (B) Representative gating strategy of human peripheral blood Tregs (CD4 ${ }^{+} \mathrm{CD} 3^{+} \mathrm{CD} 25^{+} \mathrm{CD} 127^{10-}$ $\left.\mathrm{FOXP3}^{+}\right)$. (C) Representative gating strategy for CD5 analysis. (D) Representative gating strategy and quantification of ICOS expression on $\mathrm{CD}^{10}$ and $\mathrm{CD}^{\mathrm{hi}}$ Tregs. (E) Representative expression of GITR, CTLA-4, and FOXP3 in $\mathrm{CD}^{10}$ and $C D 5^{\text {hi }}$ Tregs. (F) Quantification of FOXP3, GITR, and CTLA-4 expression. Significance was determined by Mann-Whitney $U$ test ( $\mathbf{D}$ and $\mathbf{F})$. The mean $\pm \mathrm{SEM}$ is shown. ${ }^{* * P}$ $<0.005$ and ${ }^{* *} P<0.0005$.

Interestingly, the differences in the CTLA-4 and GITR were already present at the thymic single-positive CD4 (CD4SP) stage, which suggests that an early developmental event might shape the Treg phenotype that is segregated by CD5 expression (Figure 4C and Supplemental Figure 4F). The same proportional increase in CTLA-4 and GITR were maintained in the periphery (Supplemental Figure 4G). Stable commitment to the Treg lineage is not only dependent on Foxp3 expression, but it also requires demethylation of key Treg genes in response to strong TCR signaling (41). Therefore, we sought to determine if the functional potential of Tregs is established during thymic selection and could be explained by the degree of demethylation of Treg signature genes Foxp3, Ctla4, and Tnfrsf18 (GITR). To this end, we examined CpG methylation of Treg associate genes by bisulfite sequencing of DNA isolated from $\mathrm{CD}^{\text {hi }}$ and $C D 5^{\text {lo }}$ splenic Tregs. The Foxp3 intron 1 region of $C D 5^{\text {hi }}$ and $C D 5^{\text {lo }}$ Tregs displayed nearly complete hypomethylation - 97\% and 92\%, respectively (Supplemental Figure $4 \mathrm{H}$ ). Similarly, both Treg subsets were mostly hypomethylated in the Ctla4 exon 2 region (Supplemental Figure 4I). For the $4 \mathrm{CpG}$ positions of Tnfrsf18 (GITR) exon 5, 88.6\% were hypomethylated for CD5 ${ }^{\text {hi }}$ Tregs, whereas $76.7 \%$ were hypomethylated for the CD5 ${ }^{\text {lo }}$ Tregs (Supplemental Figure $4 \mathrm{~J}$ ). Overall, the level of methylation was largely similar between $C D 5^{\text {hi }}$ and $C D 5^{\text {lo }}$ Tregs, suggesting that they are both stably committed to the Treg lineage. Interestingly, the differences in GITR and CTLA-4 expression were most pronounced in thymus and autoimmune tissue (pancreas) -2 tissue sites where Tregs are more likely to come in contact with cognate self-antigen. These observations suggest that key Treg functional genes are under continuous regulation immediately downstream of TCR signaling.

Level of CD5 expression on human Tregs correlates with functional phenotype. Although Treg-based therapies hold great promise for treatment of autoimmunity, it is unclear whether a general expansion of the Treg population in patients with autoimmunity is an efficient or effective approach. It is more likely that therapies focused on tissue antigen-specific Tregs would be more efficacious, as the cells could home to the appropriate tissue site and exert local, robust suppression $(42,43)$. Unfortunately, isolation of sufficient numbers of human antigen-specific Tregs is a challenging prospect; therefore, the use of alternative markers associated with functional self-reactive Tregs should be explored. 

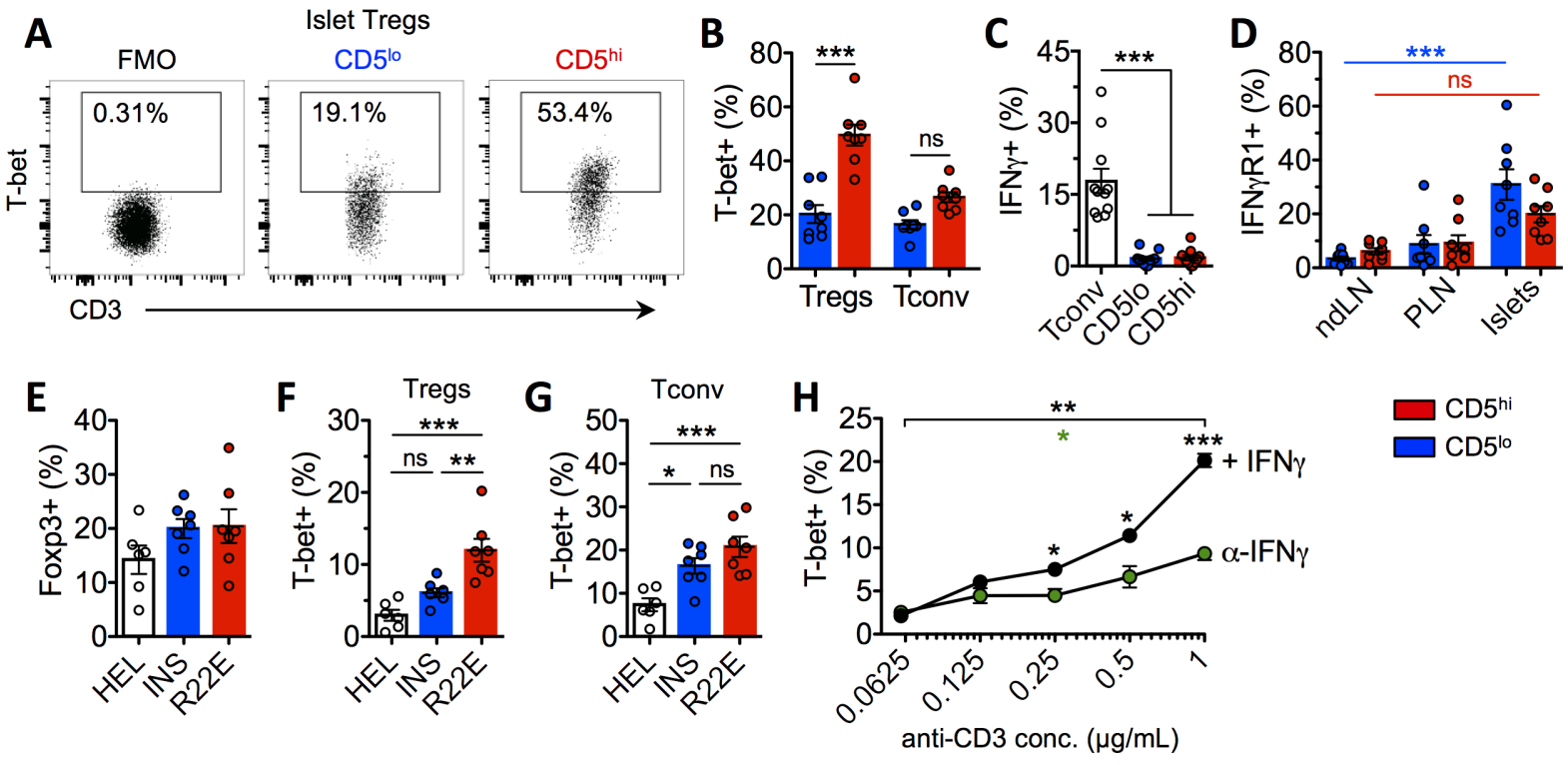

Figure 6. Strength of TCR signal regulates level of T-bet expression in Tregs. (A) Representative flow plots of T-bet expression in islet-infiltrating CD5 and $\mathrm{CD}^{10}$ Tregs (CD4+CD3+Foxp3 $3^{+}$). Fluorescence minus 1 (FMO; minus T-bet staining) was used to set T-bet gating. (B) T-bet expression in islet-infiltrating Tregs (CD4 ${ }^{+} \mathrm{CD}^{+} \mathrm{Foxp3}^{+}$) and Tconvs (CD4 $\left.{ }^{+} \mathrm{CD}^{+}{ }^{+} \mathrm{Foxp3}^{-}\right)$. An average of 8 mice from 2 experiments is shown. (C) Relative expression of IFN $\gamma$ by islet infiltrating $\mathrm{CD} 4^{+} \mathrm{Foxp}^{-}$Tconvs and $\mathrm{CD} 4^{+}{ }^{+} \mathrm{Foxp3}^{+} \mathrm{CD}^{\text {hi }}$ or $\mathrm{CD}^{10}$ Tregs after $5 \mathrm{hr}$ ex vivo stimulation with PMA + ionomycin. An average of 11 mice from 3 experiments is shown. (D) Cell surface expression of IFN $\gamma \mathrm{R} 1$ on islet-infiltrating Tregs. An average of 8 mice from 2 experiments is shown. (E-G) Analysis of inguinal draining LNs from 12-4.1 transgenic mice 12 days after immunization with InsB9-23 (INS), InsB9-23(R22E), or HEL11-25 peptide emulsified in IFA. An average of 6-7 mice from 4 experiments is shown. (E) Frequency of Tregs (CD4+CD3+Foxp3 $3^{+}$). (F) Frequency of T-bet-expressing Tregs. (G) Frequency of T-bet-expressing Tconvs. (H) TCR-mediated T-bet induction in vitro. T-bet expression was induced in CD4+CD3+Foxp3+ Tregs sorted from the spleens of 8- to 14 -week-old NOD mice by stimulating the cells for 48 hours with 1,000 U IL-2, $1 \mu \mathrm{g} / \mathrm{ml}$ plate-bound anti-CD28, and serial dilutions of plate-bound anti-CD3. Each culture also received either $25 \mathrm{ng} / \mathrm{ml} \mathrm{IFN}(+\mathrm{IFN} \gamma)$ or $2 \mu \mathrm{g} / \mathrm{ml}$ anti-IFN $\gamma(\alpha-$ IFN $\gamma)$. Analysis is gated on CD4 $4^{+}$CD3 ${ }^{+}$Foxp $3^{+}$T cells. An average of 3 mice from 1 representative experiment is shown. Significance was determined by 1-way ANOVA with Bonferroni's multiple-comparisons test (B-G) and 1-tailed paired $t$ test $(\mathbf{H})$. The mean \pm SEM is shown. $(P>0.05),{ }^{*} P<0.05,{ }^{* *} P<0.005,{ }^{* *} P<0.0005$.

We therefore sought to address whether human Tregs with an increased functional profile could similarly be identified based on the level of CD5 expression. Freshly isolated PBMCs were composed of $19 \%-34 \% \mathrm{CD}^{+} \mathrm{CD}^{+} \mathrm{T}$ cells (Figure $5 \mathrm{~A}$ ), with $\mathrm{CD} 25^{+} \mathrm{FOXP} 3{ }^{+} \mathrm{CD} 127^{\text {lo }}$ Tregs accounting for $3.2 \%-$ $5.5 \%$ of the total $\mathrm{CD} 4^{+} \mathrm{T}$ cell population (Figure $5 \mathrm{~B}$ ). In order to effectively segregate Tregs based on CD5 expression, we interrogated the top $35 \%\left(C D 5^{\text {hi }}\right)$ and bottom $35 \%\left(C D 5^{10}\right)$ of the population (Figure $5 \mathrm{C}$ ). Compared with $\mathrm{CD} 5^{\text {lo }}$ counterparts, $\mathrm{CD} 5^{\text {hi }}$ Tregs showed significantly increased expression of ICOS, FOXP3, GITR, and CTLA-4 (Figure 5, D-F). Therefore, CD5 expression correlates with an increased functional profile in human Tregs.

TCR affinity drives T-bet expression in Tregs. It is clear that both cell intrinsic (TCR) and extrinsic (inflammatory milieu) factors affect Treg function, but to what degree each is responsible for modulating Treg functional potential is unknown. Local inflammatory cues direct Tregs to take on distinct phenotypic changes and upregulate $\mathrm{T}$ helper lineage transcription factors that mirror that of their target effector subset (44-46). Several groups have described cotranscription factor expression by Tregs as being necessary for their ability to suppress specific $\mathrm{T}$ helper lineages (32, 44-47). Explicitly, coexpression of Foxp3 and T-bet by Tregs is indispensable for their ability to prevent autoimmune diabetes (32). In line with this idea, RNA-seq analysis revealed that functionally superior CD5 $5^{\text {hi }}$ islet Tregs exhibited an enhanced T-bet program (Figure $3 \mathrm{~F}$ ). At the protein level, we observed a significant increase in T-bet expression in the inflammatory tissue site, which was more pronounced in the $\mathrm{CD} 5^{\text {hi }}$ Treg subset $\left(20 \% \mathrm{~T}_{\text {-bet }}{ }^{+}\right.$in $\mathrm{CD} 5^{\text {lo }} \mathrm{vs}$. $50 \%$ in $\mathrm{CD} 5^{\text {hi }}$ ) (Figure 6, A and B). Notably, the dramatic difference in T-bet expression between CD $5^{\text {hi }}$ and $\mathrm{CD} 5^{\text {10 }} \mathrm{T}$ cells was mainly seen in the Treg compartment, with a near 3-fold difference, while the difference between islet-infiltrating $\mathrm{CD} 5^{\text {hi }}$ and $C D 5^{\text {lo }}$ Tconv subsets was substantially less significant $(16 \%$ vs. $27 \%$, Figure $6 \mathrm{~B}$ ). Although, recent studies have shown that T-bet expression can be associated with Treg instability characterized by expression of IFN $\gamma(48)$, both Treg subsets expressed similar and mostly negligible levels of IFN $\gamma$ after in vitro restimulation (Figure 6C). 
The increase in T-bet in CD5 ${ }^{\text {hi }}$ Tregs was hard to reconcile with reduced IFN $\gamma$ responsiveness (Figure 3G). Several genes downstream of IFN $\gamma$ signaling were downregulated in CD5 ${ }^{\text {hi }}$ Tregs, including Socs1, Stat1, H2-Aa, and Txk (Figure 3G). The decrease in the IFN $\gamma$ response appeared to be regulated at the receptor level, as mRNA expression of both IFN $\gamma \mathrm{R} 1$ and $-\mathrm{R} 2$ were reduced in $\mathrm{CD} 5^{\text {hi }}$ Tregs, which was verified at the protein level for IFN $\gamma \mathrm{R} 1$ (Figure $3 \mathrm{G}$ and Figure 6D). These results suggested a dominant role for TCR in regulating T-bet expression in Tregs.

In order to dissect the contribution of TCR signaling in driving T-bet expression in Tregs, we immunized insulin-specific 12-4.1 TCR transgenic (Tg) mice with strong and weak agonist insulin peptides. The binding of insulin self-peptide B9-23 (InsB9-23) to MHC I-A ${ }^{\mathrm{g} 7}$ is unstable in the target register and represents a weak agonist for TCR stimulation (49). The modified peptide R22E, on the other hand, forms a more stable peptide-MHC complex and acts as a strong agonist for insulin-specific T cells (50). Immunization of NOD mice with the strong agonist has previously been shown to protect mice from diabetes development, presumably through induction of insulin-specific Tregs (50). We hypothesized that the protection was also dependent on the level of T-bet induced by the strong agonist. Mice (12-4.1 TCR Tg) were immunized s.c. with $100 \mu \mathrm{g}$ of peptide emulsified in incomplete Freund's adjuvant (IFA) and analyzed 12 days later. While administration of hen egg lysozyme I-A ${ }^{97}$ epitope (HEL) had no effect on Treg frequencies, both InsB9-23 and the strong agonist R22E promoted a modest increase in Foxp $3^{+} \mathrm{T}$ cells (Figure 6E). Importantly, T-bet expression was induced to a higher level in R22E-treated mice than InsB9-23-treated mice (Figure 6F). Consistent with our observations in polyclonal NOD mice (Figure 6B), T-bet expression in Tconvs seemed to be less dependent on TCR signaling strength, as treatment with either R22E or InsB923 resulted in similar expression of T-bet (Figure 6G). Taken together, these data suggest that TCR signal strength is important for induction of the T-bet program in Tregs.

To further elucidate the relative contribution of TCR signaling strength and IFN $\gamma$ in inducing T-bet expression in Tregs, we stimulated purified Tregs with serial dilutions of anti-CD3 in vitro in the presence or absence of IFN $\gamma$. In an inflammatory environment $(25 \mathrm{ng} / \mathrm{ml}$ IFN $\gamma)$, strong TCR stimulation $(1 \mu \mathrm{g} / \mathrm{ml}$ anti-CD3) led to upregulation of T-bet in about $20 \%$ of Tregs (Figure $6 \mathrm{H}$ ). As the strength of TCR stimulation decreased, there was a proportional decrease in the frequency of T-bet-expressing Tregs (Figure $6 \mathrm{H}$ ), indicating that the strength of TCR signal perceived by Tregs within the inflammatory context regulates T-bet expression. Importantly, in the absence of IFN $\gamma$, assured by the addition of the IFN $\gamma$-blocking antibody, we observed a reduced but significant level of T-bet induction (Figure 6H). Taken together, these data indicate that the enhanced protective capacity of $\mathrm{CD} 5^{\text {hi }}$ Tregs in vivo relies on TCR-driven T-bet expression, necessary for their ability to control pathogenic Th1 Teffs.

\section{Discussion}

In this study, we addressed the importance of TCR self-reactivity for optimal Treg function. A number of studies have focused on the role of TCR affinity in Treg development, with limited investigation into its role after thymic selection. Through the use of conditional TCR $\alpha$-KO mice, it was recently shown that TCR is essential and is continually required for Treg function; however, it is still unclear whether the level of TCR signaling is proportional to Treg function (17). Several approaches have utilized surrogate markers to distinguish Treg populations with various levels of self-reactivity $(22,51)$. A more recent study used a combination of 3 surface markers to differentiate a population of functional Tregs in models of colitis and immune homeostasis (GITR, CD25, PD-1) (51). The authors observed that GITR, CD25, and PD-1 triple-positive Tregs expressed higher levels of Nur77GFP and CD5 and selectively controlled immune homeostasis, whereas triple-negative Tregs selectively limited colitis development. While the study brought to light the potential importance of TCR self-reactivity in driving specific Treg functions, the cell-surface markers used to identify these Treg subsets (GITR, CD25, PD-1) are dynamic in their level of expression and might constitute Treg populations at a different level of activation. Unlike the previous studies, we used a more direct approach for evaluating TCR self-reactivity in Tregs by using a single, relatively stable indicator of TCR self-reactivity - CD5. Our approach showed that $\mathrm{CD}^{\text {hi }}$ and $\mathrm{CD}^{\text {lo }}$ Tregs were able to efficiently control $\mathrm{T}$ cell homeostasis, suggesting that self-reactivity is not important for regulation of immune homeostasis. Most importantly, we also compared Tregs isolated directly from a target tissue, at the site of focused antigen-reactivity, rather than from a lymphoid organ with multitissue TCR specificities. We observed that CD $5^{\text {hi }}$ Tregs, or highly self-reactive Tregs, exhibited a more functional phenotype and were more protective in tissue-specific autoimmunity. 
The superior functional potential of $C D 5^{\text {hi }}$ tissue-infiltrating Tregs was associated with an increase in Treg signature genes: Tigit, Lag3, Ctla4, GITR, IL-10, Areg, and Icos (Figure 3E and Figure 4). Interestingly, at the protein level, ICOS expression was restricted to islet-infiltrating CD5 ${ }^{\text {hi }}$ Tregs (Figure 4D). ICOS has previously been shown to identify a highly functional islet-infiltrating Treg population, which preferentially upregulates CXCR3, suggesting an important role for TCR in driving the program necessary for regulation of Th1 islet autoimmunity $(32,52,53)$.

TCR signaling during thymocyte development is mediated by continuous sampling of multiple self-ligands, as cells progress through positive and negative selection. It is likely that CD5 expression is proportional to the net overall signal received by a $\mathrm{T}$ cell in response to several ligands. Therefore, CD5 might not always indicate a level of reactivity to a single self-peptide but rather the cumulative TCR signaling received during thymic selection (21). Nevertheless, we observed a direct correlation with CD5 and TCR-induced Nur77 ${ }^{\mathrm{GFP}}$ in islet-infiltrating Tregs (Figure 1B). Presumably, Tregs infiltrating pancreatic tissue are reactive to a particular pancreatic antigen, and in that case, CD5 has a direct correlation with reactivity to self. This is supported by the observation that reactivity to an islet antigen, based on insulin tetramer binding, is associated with higher CD5 expression on islet-infiltrating Tregs (Figure 1C).

While Tregs are generally thought to exhibit higher self-reactivity than Tconvs, whether or not tissue specificity is important for Treg function remains unknown. Some studies suggest that tissue specificity is critical for recruitment of Tregs to the target tissue $(6,8,54,55)$, while others have shown that during immunization-induced CNS autoimmunity, only about half of Treg TCRs are reactive to the immunodominant self-antigen (56). It is unclear if the remaining 50\% of Tregs are not specific to tissue antigen or if perhaps they are reactive to a different, undefined antigen. Additionally, inflammatory cues alone may be sufficient to recruit Tregs, irrespective of their tissue specificity. In our studies, islet-infiltrating CD5 ${ }^{\text {lo }}$ Tregs were likewise Nur77 ${ }^{\mathrm{GFP}}$ low, indicating lower levels of TCR signaling at the tissue site. Whether these Tregs exhibit low affinity for islet antigen or whether a proportion or all of CD5 $5^{\text {lo }}$ Tregs are tissue nonspecific is unknown. In any case, our study highlights the importance of not only tissue specificity in Treg function, but also a necessity for their high self-reactivity for optimal function in an autoimmune environment.

Our study showed that the level of TCR self-reactivity alone can be used to identify superiorly functional Tregs in preventing tissue-specific autoimmunity. Treg numbers are often elevated in target tissues during autoimmunity, yet they fail to prevent tissue destruction (57-61). This suggests that, while recruitment to the site of inflammation is unperturbed, the Treg population in autoimmunity is functionally compromised. The level of CD5 expression was sufficient to divide the polyclonal Treg population into 2 functionally distinct subsets. Importantly, both populations were able to traffic to the islets (Figure $1 \mathrm{~F}$ ). Together, these observations indicate that a broad range of self-reactive Tregs are recruited to dampen an autoimmune response, but only a fraction of the population is functional. Accumulation of nonfunctional low-affinity Tregs could potentially explain why Treg numbers increase during autoimmunity but are insufficient to control the effector response.

Several groups have reported that coexpression of Foxp3 with $\mathrm{T}$ helper lineage hallmark transcription factors, such as T-bet, GATA3, and ROR $\gamma$ t, aide Tregs in their ability to suppress specific T helper subsets $(62,63)$. It was recently demonstrated that Treg-specific deletion of T-bet abrogated the ability of Tregs to prevent autoimmune diabetes in NOD mice (32). On the other hand, under some circumstances, T-bet - and subsequently IFN $\gamma$ - expression can lead to Treg instability (48, 64-66). Thus, it remains unclear whether sustained antigen exposure, and consequently continuous TCR signaling, lead to T-bet expression and subsequent Treg destabilization. The current study provides evidence to the contrary, however, as we observed T-bet expression in the absence of IFN $\gamma$ production associated with a highly functional CD5 Treg subset. Additionally, $\mathrm{CD} 5^{\mathrm{hi}}$ and $\mathrm{CD} 5^{\text {lo }}$ Tregs retained similar Foxp3 expression after transfer, displayed relatively low expression of KLRG1 ex vivo, and exhibited similar demethylation of key Treg genes (Supplemental Figure 2, E and H, and Supplemental Figure 4, E, and H-J). Overall, our data indicate that $\mathrm{CD} 5^{\text {hi }}$ Tregs are able to maintain suppressive lineage stability under inflammatory context by shifting their dependence from inflammatory cues to primarily utilize TCR signaling pathways for activation of tissue-specific responses.

Our study suggests that the level of TCR signaling is correlated with Treg functional capacity and is critical for optimal Treg-suppressive function in demanding inflammatory settings. Although we and others have shown that certain genes and pathways necessary for Treg function are directly regulated downstream of TCR activation, it is unclear whether TCR strength of signal has only an immediate effect on Treg function 
or whether a strongly perceived TCR signal during thymic development can potentiate Treg function upon a future encounter with self-antigen in the periphery (67). If such long-lasting effects do exist, they do not seem to be regulated at the DNA methylation level, since both GITR and CTLA-4 were hypomethylated in CD5 ${ }^{\text {hi }}$ and $C D 5^{\text {lo }}$ Tregs. In either case, our findings show that strong TCR signaling is critical for optimal Treg function in tissue-specific autoimmunity.

Tregs hold a great promise as a safe and long-lasting therapy for autoimmune disorders. In vitroexpanded autologous Treg transfer has recently passed a phase 1 safety trial for treatment of type 1 diabetes (68). However, therapeutic success of such approaches depends on the functional potential, persistence, and tissue-specificity of the Treg population. Previous studies in mice have shown that polyclonal Tregs from spleens of NOD mice are not protective in comparison with tissue-specific Tregs (6). Therefore, we suspect that islet antigen-specific Tregs are found at insufficient frequencies in peripheral lymphoid tissues to provide noticeable therapeutic benefit. However, in other inflammatory or autoimmune diseases where Treg tissue specificity is not absolutely required for homing to the site of inflammation, such as GVHD, selective transfer of CD5 ${ }^{\text {hi }}$ Tregs could improve the efficacy of Treg therapy based on our phenotypic analysis of human Tregs. In regards to the clinical relevance of our study for type 1 diabetes, our findings suggest that development of successful Treg therapies not only depends on Treg tissue reactivity per se, but also on the level of Treg TCR reactivity for $\beta$ cell antigens.

\section{Methods}

Human subjects. Fresh blood samples were obtained from healthy individuals as part of a Baylor College of Medicine IRB-approved study (H-33095) after obtaining written consent. PBMCs were purified by standard Ficoll-Paque gradient centrifugation and immediately analyzed. PBMCs were collected from 8 healthy donors age 23-35, composed of 4 males and 4 females.

Mice. NOD/ShiLtJ (NOD), NOD.CB17-Prkdcscid/J (NOD.scid), NOD.129P2(C)-Tcratm1Mjo/DoiJ (NOD. tcro-/-), NOD.B6-Ptprcb/6908MrkTacJ (NOD.CD45.2), NOD/ShiLt-Tg(Foxp3-EGFP/cre)1cJbs/J (NOD.Foxp3GFP), NOD.Cg-Rag1 $1^{\text {tmIMom }}$ Tg(TcraBDC12-4.1)10Jos Tg(TcrbBDC12-4.1)82Gse/J (124.1 TCR transgenic), and C57BL/6J mice were obtained directly from the Jackson Laboratory and maintained at our facility. C57BL/6-Tg(Nr4a1-EGFP/cre)820 Khog/J were originally obtained from K. Hogquist (Univeristy of Minnesota, Minneapolis, Minnesota, USA) and backcrossed on to the NOD/ShiLtJ background to generate NOD.Nur77GFP mice. All mice were housed in specific pathogen free conditions.

Assessment of diabetes. Diabetes development was monitored weekly with Diastix (Bayer), and positive readings were confirmed with Breeze2 glucometer (Bayer). Mice were considered diabetic if their blood glucose was $>400 \mathrm{mg} / \mathrm{dl}$.

Isolation of pancreatic islets. Pancreata were perfused by injecting $3 \mathrm{ml}$ collagenase IV (Worthington) through the bile duct, harvested, and placed in $2 \mathrm{ml}$ collagenase IV. Pancreata were incubated at $37^{\circ} \mathrm{C}$ for 30 minutes, after which they were washed once with $10 \mathrm{ml} \mathrm{5 \%} \mathrm{FBS/HBSS}$ and resuspended in $10 \mathrm{ml} 5 \%$ FBS/HBSS. Islets were handpicked and incubated at $37^{\circ} \mathrm{C}$ for 15 minutes in $1 \mathrm{ml}$ cell dissociation buffer (Invirogen) and further dissociated by vortexing. Cells were then washed in $10 \mathrm{ml}$ 5\% FBS/HBSS and analyzed by flow cytometry.

Diabetes adoptive transfer model. $\mathrm{CD}^{+} \mathrm{T}$ cells were sorted from infiltrated pancreatic islets of 14- to 22-week-old male or female NOD.Foxp3 ${ }^{\mathrm{GFP}}$ mice. Sorting was performed on a FACSAria Fusion (BD Biosciences). Tregs were sorted with an average purity of $97 \%$ (Supplemental Figure $2 \mathrm{~B})$. Teffs $\left(\mathrm{CD} 4^{+} \mathrm{G}-\right.$ $\left.\mathrm{FP}^{-}\right)$and Tregs $\left(\mathrm{CD}^{+} \mathrm{GFP}^{+} \mathrm{CD} 5^{\mathrm{hi}} / \mathrm{CD}^{10}\right)$ were transferred to NOD. Tcr ${ }^{-/-}$female recipients at a 4:1 ratio (16,000 Teffs/4,000 Tregs). Mice were monitored for diabetes over a course of 30 weeks.

Homeostasis model. Single cell suspension from the spleens of 6- to 8-week-old male or female NOD. Foxp3 $^{\mathrm{GFP}}$ (CD45.1) or NOD.CD45.2 mice were stained with monoclonal antibodies against CD4, CD5, CD45RB, and CD25. Sorting was performed on a FACSAria Fusion (BD Biosciences). Tregs and Tconvs were sorted with an average purity of $98 \%$ and $97 \%$, respectively (Supplemental Figure 2, D and G). Naive Tconv $\left(\mathrm{CD} 4^{+} \mathrm{CD} 45 \mathrm{RB}^{\mathrm{Hi}} \mathrm{CD} 25^{-}\right)$alone or in combination with Tregs $\left(\mathrm{CD} 4^{+} \mathrm{GFP}^{+} \mathrm{CD} 5^{\mathrm{hi}} / \mathrm{CD} 5^{\mathrm{lo}}\right)$ were transferred to sex-matched NOD.scid recipients at a 4:1 ratio $\left(5 \times 10^{5} \mathrm{Tconv} / 1.25 \times 10^{5}\right.$ Tregs $)$. Mice were analyzed 4 weeks after transfer.

RNA-seq. RNA was isolated from $\mathrm{CD}^{+} \mathrm{T}$ cells sorted from single cell suspensions of the pancreatic islets and spleens of NOD.Foxp $3^{\mathrm{GFP}}$ mice stained with anti-CD4 and anti-CD5 antibodies. All samples were sorted with > 97\% purity. cDNA was synthesized using the SMARTer Ultra Low Input RNA Kit (Clontech). 
Library preparation was performed with the Illumina Nextera XT kit before paired-end RNA-seq using the Illumina NextSeq500 platform for 150 cycles (NextSeq500 Mid Output Kit). Sequencing reads were aligned to the mouse genome (RefSeq mm10) using TopHat Alignment (version 1.0.0; ref. 69), and gene expression was quantified by FPKM. Cufflinks Assembly \& DE (version 1.1.0; ref. 70) was used to compute differential expression between groups, with differentially expressed genes defined by $\mathrm{q}<0.05$ with Benjamini-Hochberg correction for multiple testing. Heatmaps and PCA were generated in R (version 3.2.3) using heatmap.2 from gplots package (version 2.17.0) with viridis (version 0.4.0), and ggbiplots (71). Gene ontology analysis was performed using Metascape. All original RNA-seq data were deposited in the NCBI's Gene Expression Omnibus database (GEO GSE102231).

In vivo T-bet induction. Six- to 10-week-old female 12-4.1 TCR Tg mice were injected s.c. on both hind flanks with a total of $100 \mu \mathrm{g}$ peptide emulsified in IFA.

Peptides. Insulin B:9-23 (C19A) (SHLVEALYLVAGERG; weak agonist peptide), insulin B:9-23 (R22E, C19A) (SHLVEALYLVAGEEG; strong agonist peptide), and HEL:11-25 (AMKRHGLDNYRGYSL; irrelevant peptide control) were used in tolerogenic immunizations of 12-4.1 TCR Tg mice. Mice were analyzed 12 days after injection.

In vitro T-bet induction. Tregs $\left(\mathrm{CD}^{+} \mathrm{GFP}^{+} / \mathrm{CD} 25^{+}\right)$from NOD.Foxp3 ${ }^{\mathrm{GFP}}$ or NOD mice were sorted by FACS with $>95 \%$ purity. Cells were stimulated in 96-well flat bottom plates coated with serial dilutions of anti-CD3 $(1 \mu \mathrm{g} / \mathrm{ml}$ to $0.06 \mu \mathrm{g} / \mathrm{ml})$ and $1 \mu \mathrm{g} / \mathrm{ml}$ anti-CD28, in $200 \mathrm{ml}$ complete RPMI supplemented with $10 \%$ FBS, $1000 \mathrm{U}$ IL-2, and either $25 \mathrm{ng} / \mathrm{ml}$ IFN $\gamma$ or $2 \mu \mathrm{g} / \mathrm{ml}$ anti-IFN $\gamma$ for 48 hours.

Flow cytometry and antibodies. Flow cytometry analyses were performed on LSRFortessa II (BD Biosciences), and data were analyzed with FlowJo software (Tree Star Inc.). Monoclonal antibodies against the following molecules were used: Foxp3 (FJK-16s) from eBioscience; Ki67 (B56) from BD Biosciences; CD3 (145-2C11), CD4 (GK1.5), CD5 (53-7.3), CD25 (PC61), CTLA-4 (UC10-4B9), CD8 (53-6.7), GITR (YGITR 765), CD45.2 (104), CD45.1 (A20), ICOS (C398.4A), and T-bet (4B10) from Biolegend. Insulin B:9-23(8G9E)/I-Ag7 tetramers were provided by the NIH tetramer core.

Bisulfite sequencing. Genomic DNA was isolated with a Zymo Research gDNA isolation kit (D4064) from male NOD.Foxp $3^{\mathrm{GFP}}$ splenic Tregs (top $35 \%$ ) $\mathrm{CD} 4^{+} \mathrm{GFP}^{+} \mathrm{CD} 5^{\text {hi }}$ and (bottom $35 \%$ ) $\mathrm{CD}^{+} \mathrm{GFP}^{+} \mathrm{C}$ $\mathrm{D}^{\text {lo }}$ that were isolated by FACS. Primers for bisulfite sequencing were designed for Tnfrsf 18 exon 5 (forward, 5' - GAGGTGTAGTTGTTAGTTGAGGATGT - 3') (reverse, 5' - AACCCCTACTCTCACCAAAAATATAA - 3'), Ctla4 exon 2 (forward, 5' - TGGTGTTGGTTAGTAGTTATGGTGT - 3') (reverse, 5' - AAATTCCACCTTACAAAAATACAATC - 3'), and Foxp3 intron 1 (forward, 5' - GAGGATTTGAATTGGATATGGTTTG - 3') (reverse, 5' - CAACCTTAAACCCCTCTAACATC - 3'), based on previously published primer sequences $(41,72)$. Sodium bisulfite treatment of the extracted DNA was carried out with a Zymo Research EZ DNA Methylation kit (catalog 11-335). Obtained PCR fragments were subcloned into shuttle vectors with the Zero Blunt TOPO Cloning kit (K280020, Invitrogen). Plasmid DNA isolated from single bacterial colonies was sequenced by Sanger sequencing. Sequences were analyzed using the BISMA web tool (73).

Data availability. All data that support the findings of this study are available from the corresponding author upon request.

Statistics. Diabetes incidence curves were compared using the log-rank test. Correlations were calculated by Pearson correlation. Comparisons of more than 2 groups were done using 1-way ANOVA with Bonferroni's multiple-comparisons test. Comparisons between 2 groups were done using the Mann-Whitney nonparametric test unless otherwise specified. Bars represent mean \pm SEM. $P \leq 0.05$ was considered statistically significant $\left({ }^{* *} P \leq 0.0005,{ }^{* *} P \leq 0.005,{ }^{*} P \leq 0.05\right)$. Statistical analyses were performed using Prism.

Study approval. The present animal studies were approved by the Baylor College of Medicine IACUC. The present human studies were approved by Baylor College of Medicine IRB, under H-33095 protocol. Subjects provided informed consent prior to participation in the study.

\section{Author contributions}

Conceptualization was contributed by MLS, MLB, and M. Bettini; methodology was contributed by MLS, MAS, IS, GM, M. Borowiak, MLB, and M. Bettini; investigation was contributed by MLS, MAS, SB, IS, TL, and M. Bettini; writing of the original draft was contributed by MLS; reviewing and editing of the manuscript were contributed by MLS, TL, GM, IS, MLB, and M. Bettini; GM, M. Borowiak, MLB, and $\mathrm{M}$. Bettini were resources; and MLB and M. Bettini provided supervision. 


\section{Acknowledgments}

We thank Richard (Aaron) Cox and Dario A.A. Vignali for helpful discussions; Kevin Peng and Katherine Kushner for help with data collection; the BCM Cytometry and Cell Sorting Core for assistance with cell sorting; Gretchen Diehl for providing C57BL/6 mice; Justin Gunesch, Chivon McMullen Jackson, and Emily Mace for providing human blood samples; and the NIH tetramer core for providing tetramer reagents. We would like to thank the blood donors for their contribution to this work. This work was supported by the NIH (AI125301-01A1), ADA (7-14-JF-07), NIH T32 AI053831 Immunology Scientist Training Grant to MLS, AAI Career Development Fellowship to IS, and The Robert and Janice McNair Foundation.

Address correspondence to: Maria Bettini, Department of Pediatrics, Texas Children's Hospital, 6701 Fannin St., Suite 1020, Houston, Texas 77030, USA. Phone: 832.824.0712. Email: maria.bettini@bcm.edu.

IS's present address is: Institute for Medical Microbiology, Immunology and Hygiene, School of Medicine, Technical University of Munich, Munich, Germany.

1. Vignali DA, Collison LW, Workman CJ. How regulatory T cells work. Nat Rev Immunol. 2008;8(7):523-532

2. Burzyn D, et al. A special population of regulatory T cells potentiates muscle repair. Cell. 2013;155(6):1282-1295.

3. Arpaia N, et al. A Distinct Function of Regulatory T Cells in Tissue Protection. Cell. 2015;162(5):1078-1089.

4. Sawant DV, Vignali DA. Once a Treg, always a Treg? Immunol Rev. 2014;259(1):173-191.

5. Lathrop SK, Santacruz NA, Pham D, Luo J, Hsieh CS. Antigen-specific peripheral shaping of the natural regulatory T cell population. J Exp Med. 2008;205(13):3105-3117.

6. Tang Q, et al. In vitro-expanded antigen-specific regulatory T cells suppress autoimmune diabetes. J Exp Med. 2004;199(11):1455-1465.

7. Zhang H, Podojil JR, Chang J, Luo X, Miller SD. TGF-beta-induced myelin peptide-specific regulatory T cells mediate antigen-specific suppression of induction of experimental autoimmune encephalomyelitis. J Immunol. 2010;184(12):6629-6636.

8. Killebrew JR, Perdue N, Kwan A, Thornton AM, Shevach EM, Campbell DJ. A self-reactive TCR drives the development of Foxp3+ regulatory T cells that prevent autoimmune disease. J Immunol. 2011;187(2):861-869.

9. Levine AG, et al. Suppression of lethal autoimmunity by regulatory T cells with a single TCR specificity. J Exp Med. 2017;214(3):609-622.

10. Hsieh CS, Zheng Y, Liang Y, Fontenot JD, Rudensky AY. An intersection between the self-reactive regulatory and nonregulatory T cell receptor repertoires. Nat Immunol. 2006;7(4):401-410.

11. Hood JD, Zarnitsyna VI, Zhu C, Evavold BD. Regulatory and T Effector Cells Have Overlapping Low to High Ranges in TCR Affinities for Self during Demyelinating Disease. J Immunol. 2015;195(9):4162-4170.

12. Lee HM, Bautista JL, Scott-Browne J, Mohan JF, Hsieh CS. A broad range of self-reactivity drives thymic regulatory T cell selection to limit responses to self. Immunity. 2012;37(3):475-486.

13. Moran AE, Hogquist KA. T-cell receptor affinity in thymic development. Immunology. 2012;135(4):261-267.

14. Pacholczyk R, Kern J. The T-cell receptor repertoire of regulatory T cells. Immunology. 2008;125(4):450-458.

15. Bautista JL, et al. Intraclonal competition limits the fate determination of regulatory $\mathrm{T}$ cells in the thymus. Nat Immunol. 2009;10(6):610-617.

16. Leung MW, Shen S, Lafaille JJ. TCR-dependent differentiation of thymic Foxp3+ cells is limited to small clonal sizes. J Exp Med. 2009;206(10):2121-2130.

17. Levine AG, Arvey A, Jin W, Rudensky AY. Continuous requirement for the TCR in regulatory T cell function. Nat Immunol. 2014;15(11):1070-1078.

18. Bettini M, et al. TCR affinity and tolerance mechanisms converge to shape T cell diabetogenic potential. J Immunol. 2014;193(2):571-579.

19. Azzam HS, Grinberg A, Lui K, Shen H, Shores EW, Love PE. CD5 expression is developmentally regulated by T cell receptor (TCR) signals and TCR avidity. J Exp Med. 1998;188(12):2301-2311.

20. Mandl JN, Monteiro JP, Vrisekoop N, Germain RN. T cell-positive selection uses self-ligand binding strength to optimize repertoire recognition of foreign antigens. Immunity. 2013;38(2):263-274.

21. Hogquist KA, Jameson SC. The self-obsession of T cells: how TCR signaling thresholds affect fate 'decisions' and effector function. Nat Immunol. 2014;15(9):815-823.

22. Delpoux A, et al. TCR signaling events are required for maintaining CD4 regulatory T cell numbers and suppressive capacities in the periphery. J Immunol. 2014;193(12):5914-5923.

23. Liu Z, Gerner MY, Van Panhuys N, Levine AG, Rudensky AY, Germain RN. Immune homeostasis enforced by co-localized effector and regulatory T cells. Nature. 2015;528(7581):225-230

24. Herman AE, Freeman GJ, Mathis D, Benoist C. CD4+CD25+ T regulatory cells dependent on ICOS promote regulation of effector cells in the prediabetic lesion. J Exp Med. 2004;199(11):1479-1489.

25. Zhang N, et al. Regulatory T cells sequentially migrate from inflamed tissues to draining lymph nodes to suppress the alloimmune response. Immunity. 2009;30(3):458-469.

26. Schmidt EM, et al. Ctla-4 controls regulatory T cell peripheral homeostasis and is required for suppression of pancreatic islet autoimmunity. J Immunol. 2009;182(1):274-282. 
27. Obata Y, et al. The epigenetic regulator Uhrf1 facilitates the proliferation and maturation of colonic regulatory $\mathrm{T}$ cells. Nat Immunol. 2014;15(6):571-579.

28. Forster F, et al. Guanylate binding protein 1-mediated interaction of T cell antigen receptor signaling with the cytoskeleton. J Immunol. 2014;192(2):771-781.

29. Demetriou M, Granovsky M, Quaggin S, Dennis JW. Negative regulation of T-cell activation and autoimmunity by Mgat5 N-glycosylation. Nature. 2001;409(6821):733-739.

30. Fassett MS, Jiang W, D'Alise AM, Mathis D, Benoist C. Nuclear receptor Nr4a1 modulates both regulatory T-cell (Treg) differentiation and clonal deletion. Proc Natl Acad Sci USA. 2012;109(10):3891-3896.

31. Levine AG, et al. Stability and function of regulatory T cells expressing the transcription factor T-bet. Nature. 2017;546(7658):421-425.

32. Tan TG, Mathis D, Benoist C. Singular role for T-BET+CXCR3+ regulatory T cells in protection from autoimmune diabetes. Proc Natl Acad Sci USA. 2016;113(49):14103-14108.

33. Koch MA, Thomas KR, Perdue NR, Smigiel KS, Srivastava S, Campbell DJ. T-bet(+) Treg cells undergo abortive Th1 cell differentiation due to impaired expression of IL-12 receptor $\beta 2$. Immunity. 2012;37(3):501-510.

34. Ronchetti S, et al. Glucocorticoid-induced tumour necrosis factor receptor-related protein: a key marker of functional regulatory T cells. J Immunol Res. 2015;2015:171520.

35. Walker LS. Treg and CTLA-4: two intertwining pathways to immune tolerance. J Autoimmun. 2013;45:49-57.

36. Cheng G, Yu A, Malek TR. T-cell tolerance and the multi-functional role of IL-2R signaling in T-regulatory cells. Immunol Rev. 2011;241(1):63-76.

37. Morikawa H, Sakaguchi S. Genetic and epigenetic basis of Treg cell development and function: from a FoxP3-centered view to an epigenome-defined view of natural Treg cells. Immunol Rev. 2014;259(1):192-205.

38. Rudensky AY. Regulatory T cells and Foxp3. Immunol Rev. 2011;241(1):260-268.

39. Cheng G, Yuan X, Tsai MS, Podack ER, Yu A, Malek TR. IL-2 receptor signaling is essential for the development of Klrg1+ terminally differentiated T regulatory cells. J Immunol. 2012;189(4):1780-1791.

40. Kornete M, Mason E, Istomine R, Piccirillo CA. KLRG1 expression identifies short-lived Foxp3+ Treg effector cells with functional plasticity in islets of NOD mice. Autoimmunity. 2017;50(6):354-362.

41. Ohkura N, et al. T cell receptor stimulation-induced epigenetic changes and Foxp3 expression are independent and complementary events required for Treg cell development. Immunity. 2012;37(5):785-799.

42. Brusko TM, Putnam AL, Bluestone JA. Human regulatory T cells: role in autoimmune disease and therapeutic opportunities. Immunol Rev. 2008;223:371-390.

43. Bluestone JA, Bour-Jordan H, Cheng M, Anderson M. T cells in the control of organ-specific autoimmunity. J Clin Invest. 2015;125(6):2250-2260.

44. Koch MA, Tucker-Heard G, Perdue NR, Killebrew JR, Urdahl KB, Campbell DJ. The transcription factor T-bet controls regulatory $\mathrm{T}$ cell homeostasis and function during type 1 inflammation. Nat Immunol. 2009;10(6):595-602.

45. Chaudhry A, et al. CD4+ regulatory T cells control TH17 responses in a Stat3-dependent manner. Science. 2009;326(5955):986-991.

46. Zheng Y, et al. Regulatory T-cell suppressor program co-opts transcription factor IRF4 to control T(H)2 responses. Nature. 2009;458(7236):351-356.

47. Yu F, Sharma S, Edwards J, Feigenbaum L, Zhu J. Dynamic expression of transcription factors T-bet and GATA-3 by regulatory T cells maintains immunotolerance. Nat Immunol. 2015;16(2):197-206.

48. Overacre-Delgoffe AE, et al. Interferon- $\gamma$ Drives Treg Fragility to Promote Anti-tumor Immunity. Cell. 2017;169(6):1130-1141.e11.

49. Levisetti MG, Suri A, Petzold SJ, Unanue ER. The insulin-specific T cells of nonobese diabetic mice recognize a weak MHC-binding segment in more than one form. J Immunol. 2007;178(10):6051-6057.

50. Daniel C, Weigmann B, Bronson R, von Boehmer H. Prevention of type 1 diabetes in mice by tolerogenic vaccination with a strong agonist insulin mimetope. J Exp Med. 2011;208(7):1501-1510.

51. Wyss L, et al. Affinity for self antigen selects Treg cells with distinct functional properties. Nat Immunol. 2016;17(9):1093-1101.

52. Kornete M, Sgouroudis E, Piccirillo CA. ICOS-dependent homeostasis and function of Foxp3+ regulatory T cells in islets of nonobese diabetic mice. J Immunol. 2012;188(3):1064-1074.

53. Kornete M, Mason ES, Girouard J, Lafferty EI, Qureshi S, Piccirillo CA. Th1-Like ICOS+ Foxp3+ Treg Cells Preferentially Express CXCR3 and Home to $\beta$-Islets during Pre-Diabetes in BDC2.5 NOD Mice. PLoS One. 2015;10(5):e0126311.

54. Tarbell KV, Yamazaki S, Olson K, Toy P, Steinman RM. CD25+ CD4+ T cells, expanded with dendritic cells presenting a single autoantigenic peptide, suppress autoimmune diabetes. J Exp Med. 2004;199(11):1467-1477.

55. Dudda JC, Perdue N, Bachtanian E, Campbell DJ. Foxp3+ regulatory T cells maintain immune homeostasis in the skin. $J$ Exp Med. 2008;205(7):1559-1565.

56. Liu X, et al. T cell receptor CDR3 sequence but not recognition characteristics distinguish autoreactive effector and Foxp3(+) regulatory T cells. Immunity. 2009;31(6):909-920

57. Makita S, et al. CD4+CD25bright T cells in human intestinal lamina propria as regulatory cells. J Immunol. 2004;173(5):3119-3130.

58. Feger U, Luther C, Poeschel S, Melms A, Tolosa E, Wiendl H. Increased frequency of CD4+ CD25+ regulatory T cells in the cerebrospinal fluid but not in the blood of multiple sclerosis patients. Clin Exp Immunol. 2007;147(3):412-418.

59. Cao D, Malmström V, Baecher-Allan C, Hafler D, Klareskog L, Trollmo C. Isolation and functional characterization of regulatory CD25brightCD4+ T cells from the target organ of patients with rheumatoid arthritis. Eur J Immunol. 2003;33(1):215-223.

60. Korn T, et al. Myelin-specific regulatory T cells accumulate in the CNS but fail to control autoimmune inflammation. Nat Med. 2007;13(4):423-431.

61. Tang $\mathrm{Q}$, et al. Central role of defective interleukin-2 production in the triggering of islet autoimmune destruction. Immunity. 2008;28(5):687-697.

62. Campbell DJ, Koch MA. Phenotypical and functional specialization of FOXP3+ regulatory T cells. Nat Rev Immunol. 2011;11(2):119-130.

63. Duhen T, Duhen R, Lanzavecchia A, Sallusto F, Campbell DJ. Functionally distinct subsets of human FOXP3+ Treg cells that phenotypically mirror effector Th cells. Blood. 2012;119(19):4430-4440. 
64. Zhou X, Bailey-Bucktrout S, Jeker LT, Bluestone JA. Plasticity of CD4(+) FoxP3(+) T cells. Curr Opin Immunol. 2009;21(3):281-285

65. Bailey-Bucktrout SL, et al. Self-antigen-driven activation induces instability of regulatory T cells during an inflammatory autoimmune response. Immunity. 2013;39(5):949-962.

66. Zhou X, et al. Instability of the transcription factor Foxp3 leads to the generation of pathogenic memory T cells in vivo. Nat Immunol. 2009;10(9):1000-1007.

67. Klein L, Kyewski B, Allen PM, Hogquist KA. Positive and negative selection of the T cell repertoire: what thymocytes see (and don't see). Nat Rev Immunol. 2014;14(6):377-391.

68. Bluestone JA, et al. Type 1 diabetes immunotherapy using polyclonal regulatory T cells. Sci Transl Med. 2015;7(315):315ra189.

69. Trapnell C, Pachter L, Salzberg SL. TopHat: discovering splice junctions with RNA-Seq. Bioinformatics. 2009;25(9):1105-1111.

70. Trapnell C, et al. Differential gene and transcript expression analysis of RNA-seq experiments with TopHat and Cufflinks. Nat Protoc. 2012;7(3):562-578.

71. Wickham H. ggplot2: elegant graphics for data analysis. New York, NY: Springer-Verlag New York; 2009.

72. Kalekar LA, et al. CD4(+) T cell anergy prevents autoimmunity and generates regulatory T cell precursors. Nat Immunol. 2016;17(3):304-314.

73. Rohde C, Zhang Y, Reinhardt R, Jeltsch A. BISMA--fast and accurate bisulfite sequencing data analysis of individual clones from unique and repetitive sequences. BMC Bioinformatics. 2010;11:230. 